Chapter 6

\title{
Pathogenesis, Clinical Diagnosis and Treatment, and Animal Models for Ckd-Mbd
}

\author{
Yan Zhang and Yoseph Asmelash Gebru \\ Additional information is available at the end of the chapter \\ http://dx.doi.org/10.5772/54238
}

\section{Introduction}

People with chronic kidney disease (CKD) develop changes in circulating blood levels of calcium and phosphorus. The kidney gradually loses the ability to remove phosphorus from the blood and cannot produce adequate amounts of active vitamin D to maintain normal levels of calcium. This occurs mainly because of decreased renal excretion of phosphate and diminished renal hydroxylation of 25-hydroxyvitamin D to calcitriol due to low expression of alpha-1-hydroxylase in the failed kidneys [1]. Further compensation to maintain normal serum calcium and phosphorus homeostasis includes increased production and release of parathyroid hormone (PTH) and potentially other phosphaturic factors, such as fibroblast growth factor-23 (FGF23) [2].

Two main complications follow to the above mentioned molecular responses namely secondary hyperparathyroidism (sHPT) and vascular calcification (VC), which occur in a high percentage of CKD patients [1]. These molecular disorders alter bone metabolism which leads to bone abnormalities including altered bone production and resorption. In turn, bony changes may result in bone deformation, bone pain, and more risks of fracture [3]. All of the above biochemical abnormalities (calcium, phosphorus, vitamin D and PTH disturbances) and vascular calcification as well as changes in bone metabolism such as variation in turnover and bone mineralization can be included under the descriptions for CKD-associated mineral and bone disorders (CKD-MBD) [4].

This review explains the main pathological causes and mechanisms of CKD-MBD and the possible animal models for basic research on this disease. It also describes some clinically applicable diagnosis techniques and treatment methods with their advantages and side effects for CKD-MBD. 


\section{Potential pathological mechanisms}

\section{1. sHPT-related bone disorders}

The first changes that usually occur with the decline of renal function involve the deficiency of active vitamin $\mathrm{D}$ and decrease in phosphorus excretion by the remaining functional nephrons [5]. In CKD, the failed kidney is inefficient in alpha-1-hydroxylase expression resulting in low synthesis of calcitriol. Simultaneously the kidney with lower function has reduced ability to reabsorb calcium from urine [6]. Therefore, low serum calcium level, high serum phosphorus level and impaired renal 1, 25-dihydroxyvitamin D synthesis with attendant reduction in serum calcitriol concentration and decrease in vitamin $\mathrm{D}$ receptor expression in the parathyroid glands contribute to excess PTH secretion in patients with CKD [7].

PTH strongly influences the exchange of calcium to and from bone through its involvement in bone cell apoptosis mechanisms and effects on the receptor activator of NF-kappa B (RANK)/receptor activator of NF-kappa B ligand (RANKL)/osteoprotegerin (OPG) axis. Continuously elevated PTH could upregulate RANKL expression, leading to an increase in the formation rate and survival time of bone-resorbing osteoclasts and net bone loss [8,9]. Excess PTH also leads to high bone turnover, a condition characterized by accelerated rates of bone formation and bone resorption [1]. The high bone turnover due to sHPT is accompanied by about $5 \%$ (up to $10 \%$ ) lower bone mass, which is partly reversible (low mineral bone, increased remodeling space) and partly irreversible (cortical thinning) [10]. The new formed bone in the course of sHPT is structurally inferior and fragile, and carries an increased risk of fractures.

Another main molecular mechanism underlying sHPT is attributed to Klotho-fibroblast growth factor-23 (FGF-23) system. Humans with CKD experience decreased Klotho expression as early as stage $1 \mathrm{CKD}$. Klotho continues to decline as CKD progresses, causing FGF-23 resistance and provoking large FGF-23 and parathyroid hormone increases [11]. FGF-23 is a novel bone-derived hormone, in conjunction with its co-receptor, Klotho, activates FGF receptor 1 (FGFR1) and acts on the kidney to induce renal phosphate wasting and to suppress 1,25-dihydroxyvitamin $\mathrm{D}$ synthesis [12]. In patients with $\mathrm{CKD}$, circulating FGF23 levels are progressively increased to compensate for persistent phosphate retention, but this result in reduced renal production of 1, 25-dihydroxyvitamin D through suppressing $1 \alpha$-hydroxylase activity, which leads to sHPT [13].

\subsection{VC-related bone disorders}

Vascular calcification is very common in patients with CKD, appearing in 30-65\% of patients with stage 3-5 CKD, 50-80\% of patients with stage 5 CKD [14]. Calcium, a divalent cation, and phosphate, a trivalent anion, have a high binding affinity for one another and as the concentration of one or both ions increases in serum, there is an increased risk for an ionic bond to form, creating an insoluble complex which leads to vascular calcification [5]. Clinically, when the serum calcium-phosphate product exceeds $60 \mathrm{mg}^{2} / \mathrm{dl}^{2}$, widespread tissue deposition of amorphous calcium phosphate occurs [15]. 
There are a large number of promoters and inhibitors involved in vascular calcification and there are more vascular calcification inhibitors circulating in the blood under normal conditions [1]. Phosphorus is the most significant studied vascular calcification promoter which is available at higher level in patients with low renal function. Hyperphosphatemia reverses the normal process in which calcification inhibitors are down-regulated, while promoters are up-regulated [1].

On the other hand, many bone-associated proteins including osteocalcin, osteopontin and osteoprotegerin, and many bone morphogenetic proteins are involved in the process of VC [16]. Previous studies have proven that active mineralization mechanisms clearly resembling those of skeletal endochondral and membranous ossification participate in vascular calcium accumulation [17]. The findings of bone-related factors in the vasculature and the vascular calcification observed in several gene-knockout mouse models imply that CKD-MBD is an actively regulated process that may be preventable or even reversed [18]. The most striking among these mouse models is the matrix gamma-carboxyglutamic acid (Gla) protein (MGP) knockout mouse, which exhibits extensive and lethal calcification and cartilaginous metaplasia of the media of all elastic arteries as early as 2 weeks after birth $[19,20]$, indicating that this protein may be of primary importance in human vascular calcification [18].

\section{Clinical diagnosis}

\subsection{Biomarkers}

According to the standardized diagnostic criteria for CKD-MBD developed and published by the international expert consensus group, kidney disease improving global outcomes (KDIGO), monitoring serum levels of calcium, phosphorus, PTH, and alkaline phosphatase is strongly recommended, and the frequency of monitoring is based on the occurrence and extent of abnormalities as well as the rate of CKD progression [21]. Phosphorus level equal to the upper phosphorus level of $5.5 \mathrm{mg} / \mathrm{dL}$ and calcium level more than $9.5 \mathrm{mg} / \mathrm{dL}$ have been suggested to be associated with increased mortality in CKD patients [22]. The combined use of second- and third-generation PTH assays allows to measure PTH (1-84) and PTH (7-84) as PTH (7-84) interacts with distinct receptors and thereby may have important roles in the regulation of bone resorption and serum calcium concentration [23]. The frequency of measurement on alkaline phosphatases is very similar to that of PTH and can provide additional information on bone turnover. The recent more KDIGO guidelines recommend that the measurement on alkaline phosphatase levels should commence in stage 3 of CKD, and that in patients with stage 4-5 of CKD, alkaline phosphatase should be measured at least every 12 months [24].

The first measurable biomarker in urine is the decline of secreted Klotho expression (as detected by western blotting of concentrated urine samples, normalized to the same creatinine content) occurs as early as stage 1 of CKD [25], therefore, Kuro-O contends that decreased Klotho expression is the initiator of CKD-MBD pathophysiology and is potentially an early 
clinical marker of CKD [11]. The study, performed on sixty pre-dialysis patients with CKD $1-5$, showed that the changing of serum OPG level happened at the earliest time (CKD 3) and its correlation coefficient with estimated glomerular filtration rate (eGFR) and BMD of Ward's triangle was statistically high, suggesting serum OPG may be a useful biomarker for early diagnosis of CKD-MBD [26], additionally, the multivariate analysis demonstrated that OPG was associated with aortic stiffness in patients with CKD stages 3-4, indicating OPG is also a marker to evaluate the cardiomyocyte dysfunction of CKD-MBD [27].

\subsection{Imaging}

Histomorphometry remains the gold standard to evaluate bone, but it is rarely performed in clinical practice. Areal measurement of bone mineral density by dual-energy x-ray absorptiometry (DEXA) is routinely performed to evaluate bone mass. However, this technique presents some limitations. In 2000, the United States National Institutes of Health defined new "quality" criteria for the diagnosis of osteoporosis in addition to decreased bone mass. Bone strength actually integrates two concepts: bone quantity and bone quality (i.e., microarchitectural organization, bone turnover, bone material properties such as mineralization, collagen traits, and micro-damage) that cannot be evaluated by DEXA. New three-dimensional, noninvasive bone-imaging techniques have thus been developed, e.g., high-resolution peripheral quantitative computed tomography (HR-pQCT). HR-pQCT allows evaluation of both volumetric density and microarchitecture in different compartments of bone [28]. Bacchetta reported for the first time an early impairment of trabecular microarchitecture in stage 2-4 CKD patients using a noninvasive bone-imaging device, HR-pQCT [29].

Physicians usually use a variety of noninvasive imaging tools to identify VC, some with merely qualitative and others with both qualitative and quantitative capabilities. Plain $x-$ rays and ultra-sonography can be used to identify macroscopic calcification of aorta and peripheral arteries, and computed tomography technologies constitute the gold standard for quantification of cardiovascular calcification [30].

\section{Clinical treatment strategy}

The clinical treatment for CKD-MBD targets the possible pathological mechanisms of mainly sHPT and VC in patients with kidney failure as treating these abnormalities will have a direct positive impact on preventing the metabolic bone disease. However, the heterogeneity of CKD-MBD makes strict protocol-driven therapeutic approaches difficult. Accordingly, considerable individualized therapy is required [31]. The followings are currently the most common and effective intervening methods.

\subsection{Phosphate binders}

In patients at stage 3-5 of CKD, multiple studies from different parts of the world have shown that higher levels of serum phosphorus have been associated with an increased relative risk of mortality [24]. Many clinical trials show that phosphate binders are effective in 
reducing serum phosphorus and PTH levels [32]. Therefore, the use of phosphate binders might be a promising and most practical strategy for the prevention of VC and SHPT which are the main pathological manifestations of the metabolic bone disease in CKD patients. The following categories of phosphate binders are being applied clinically so far:

1. Aluminum-based phosphate binders are the first type of phosphate binders to be used. They are very effective at controlling phosphorus. The most common binder of this type is aluminum hydroxide. However, aluminum has toxic effects on bone and nervous system. For this reason, aluminum-based phosphate binders are not often used much anymore [33].

2. Calcium-based phosphate binders are effective in binding phosphates and can be source of calcium. Common types of calcium-based binders include calcium acetate and calcium carbonate, both of which could cause the elevation of free calcium cation level in the gastrointestinal tract and the subsequent increase of intestinal calcium absorption [34]. The Japanese Society of Dialysis Therapy (JSDT) clinical practice guideline has recommended a higher level of these oral phosphate binders as the upper limit for clinical use [35]. These binders can also serve as calcium supplements if the calcium is low. However, if the patient is taking vitamin D supplements, he/she may already have high calcium levels, and these types of phosphate binders may provide more calcium than the normal level (i.e., excess calcium load). Therefore using calcium based phosphate binders should be accompanied with monitoring calcium levels and it should be prescribed while limiting total calcium intake.

3. Aluminum-free, calcium-free phosphate binders are newer binders that are effective at controlling phosphorus. Because they do not contain aluminum or calcium, they do not cause problems with excess aluminum or calcium load. Lanthanum carbonate is a novel non-calcium, non-aluminum phosphate-binding agent, and has been approved for clinical use in patients on hemodialysis in Japan on March in 2009 [33]. Sevelamer is a polymeric amine, which is the only non-absorbed, non-calcium-based phosphate binder currently indicated for phosphate control. The first formulation of sevelamer to be approved was sevelamer hydrochloride, while a newer formulation, sevelamer carbonate, has more recently become available [36]. Sevelamer carbonate was developed to offer phosphorus lowering while eliminating the risk of worsening metabolic acidosis associated with sevelamer hydrochloride and the consequent need to monitor for changes in serum chloride or bicarbonate levels [37].

\subsection{Vitamin D compounds}

Vitamin D analogues suppress PTH synthesis and secretion in patients with sHPT. Repletion with native vitamin D may lead to improved control of secondary hyperparathyroidism in patients with CKD which reduces the risk of bone mineral disease. It has been demonstrated that treatment with vitamin $\mathrm{D}$ analogues can decrease mortality in dialysis patients [38]. There might be some differences in clinical outcomes among vitamin D compounds with fewer calcemic and phosphatemic effects [39], such as paricalcitol, doxercalciferol, and 
maxacalcitol. Therefore, it is important for desirable active vitamin D compounds to achieve optimal vitamin D receptor (VDR) activation without inducing hypercalcemia. It is likely that the elevated calcium levels caused by calcitriol may be directly and/or indirectly responsible for the relative risk of the cardiovascular diseases that are aggravated by hypercalcemia in patient populations.

\subsection{Calcimimetics}

Calcimimetics bind to the calcium sensing receptor (CaSR) in parathyroid gland and mimic the effect of an elevated extracellular ionized calcium concentration. These molecules reduce serum levels of PTH and calcium, with a leftward shift in the set-point for calcium-regulated PTH secretion [40]. Cinacalcet is the only clinically available calcimimetic and has been shown to be a very effective therapeutic compound in the metabolic bone disease associated with CKD. Many clinical trials with cinacalcet in hemodialysis patients have shown a reduction in parathyroid hormone, calcium, phosphate and calcium $\times$ phosphate product levels, allowing far greater success in reaching therapeutic goals as recommended by international guidelines [41]. In addition to effective control of secondary hyperparathyroidism, treatment with cinacalcet may improve the mineral balance in patients with dialysis who have serum phosphate/calcium disequilibrium, and furthermore helps treating the vascular calcification as well. While, calcimimetics are not approved for use in paediatric patients with CKD and long-term data on their effects on bone, growth and biochemical parameters in children are lacking. Thus, further studies are warranted to determine the optimal strategy for controlling secondary hyperparathyroidism in the paediatric CKD population [42].

\subsection{Administering BMP-7}

One of the bone morphogenetic proteins, BMP-7, also known as osteogenic protein 1, is highly expressed in the adult kidney, and circulates in the bloodstream [43]. Therefore, it is apparent that the decrease of renal mass results in the decreased production of BMP-7, causing mineral bone disease in CKD patients [44]. One may expect an accumulation of osteoblast precursors as stimulated by PTH in CKD. While, these progenitors may be unable to differentiate mature osteoblasts because of BMP-7 deficiency considering it is important in osteoblast development and function. In this situation, the subsequent accumulation of fibrous cells could then offer an explanation for the marrow fibrosis observed in secondary hyperparathyroidism in the setting of CKD and applying BMP-7 externally can heal the disorder. There are an increasing number of recent clinical trials that provide supportive evidence for the use of BMP-7 in the treatment of fractures and bone nonunions [45]. It is not yet started to use BMP-7 as a routine clinical treatment tool except for trials in patients even though many of the studies have shown the bone healing efficacy of this molecule.

\subsection{Surgery on thyroid gland}

A surgical correction in the parathyroid gland is the final, symptomatic therapy for the most severe forms of SHPT which cannot be controlled by the above medical treatments. 
The 2009 KDIGO guideline suggested parathyroidectomy to patients who are at CKD stages 3-5 with severe hyperparathyroidism and fail to respond to medical/pharmacological therapy [46]. There are two main surgical procedures which are generally used, namely subtotal parathyroidectomy and total parathyroidectomy with immediate autotransplantation. The number and size of affected parathyroid glands are the most important factors for selecting the treatment procedure [47, 48]. Clinical studies proved that parathyroidectomy with autotransplantation from forearm was significantly effective and safe in patients in whom medical treatment had failed, particularly in terms of improving calcium and phosphate control $[49,50]$. The procedure need to be performed as early as possible to avoid the adverse, irreversible effects of prolonged hyperparathyroidism, and to improve osteoarticular symptoms. Future strategies may focus on the stimulation of apoptotic activity of hyperplastic parathyroid cells [51].

\section{Animal models}

\subsection{5/6 nephrectomy model}

Experimental model of 5/6 nephrectomy or the remnant kidney model represents one of the most used animal models of progressive renal failure by reducing nephron number, best-characterized in rats [52]. The reduction of renal mass is achieved by either infarction or surgical excision of both poles, with removal of the contra-lateral kidney. The 5/6 nephroctomy model has been found to produce serum creatinine level which is on average 2.2-fold higher than control animals, and thereafter, if without the concurrent use of vitamin $\mathrm{D}$, the phosphorus level after 8 weeks of surgery would range up to 2.6-fold higher than control animals [53]. Increased fibrosis, increased number of osteoblasts and osteoclasts as well as a mineralization defect (increased osteoid volumes and osteoid surface), those of which are typical bone changes upon sHPT, have been observed in 5/6 nephrectomy animal models [14].

The operation of 5/6 nephrectomy, combining with a diet containing $1.2 \% \mathrm{P}$ plus $0.6 \% \mathrm{Ca}$, could effectively induce sHPT in rats [54]. Additionally, the progressive partial nephrectomy with thyroparathyroidectomy (TPTx-Nx) reduced the storage modulus, which is a mechanical factor, in CKD model rats as compared with controls that underwent thyroparathyroidectomy alone (TPTx). Moreover, the TPTx-Nx rats exerted different cortical bone chemical composition and increased enzymatic crosslinks ratio and pentosidine to matrix ratio [55].

As concerned as VC associated with CKD-MBD, it can be induced in 5/6 nephrectomy rat model by feeding a high-phosphorus, high-lactose diet (1.2\% P, 1\% Ca, and 20\% lactose) after 10 weeks follow up for the reason that lactose increases calcium and phosphorus absorption in intestine [56]. 


\subsection{Electrocautery models}

In the mouse electrocautery model, CKD is induced by surgical ablation of the kidneys. This is a two-step procedure. Initially the cortex of one kidney is electrocauterized paying careful attention to avoid destroying the adrenals and the hilum of kidney. One week later, once the animals have recovered, the second kidney is nephrectomized [44]. This procedure appears to produce variable severity of CKD with blood urea levels ranging from 1.5- to 4.8-fold higher than normal animals [53]. This murine model displayed an increase in osteoblast surface and osteoid accumulation as well as increased activation frequency and increased osteoclast surface consistent with high turnover renal osteodystrophy [44]. Lund developed a standard CKD rat models by involving electrocauthery of the right kidney followed by nephroctomy of the left kidney, and found that there was a significant hyperosteoidosis produced in this model as a result of the secondary hyperparathyroidism [57].

\subsection{Adenine-contained diet}

Normally, adenine is efficiently salvaged by adenine phosphoribosyltransferase (APRT) and is present at very low level in blood and urine. APRT is involved in the conversion of adenine to adenosine monophosphate. When adenine is administered in high level, APRT activity is saturated and adenine is oxidized to 2,8-dihydroxyadenine. Adenine and 2,8-dihydroxyadenine are excreted in the urine. However, the very low solubility of 2,8-dihydroxyadenine results in its precipitation in the kidney. The accumulation of insoluble 2,8-dihydroxyadenine results in nephrolithiasis and renal failure with permanent kidney damage. Induction of chronic renal failure (CRF) in mice by dietary administration of $0.75 \%$ adenine for 4 weeks results in irreversible renal dysfunction and then CKD [53]. High-adenine feeding in rats results in the formation of crystals in the renal tubules, with subsequent tubular injury and inflammation, obstruction, and marked fibrosis [56]. Future investigations of the biochemical basis for the link between vascular calcification and bone resorption will be facilitated by the present discovery that a synthetic, $2.5 \%$ protein diet containing $0.75 \%$ adenine produces consistent and dramatic medial calcification in adult rats within just 4 weeks [58].

\subsection{Gene knockout mice}

JCK mouse is a genetic model of polycystic kidney disease. At 6 weeks of age, the mice have normal renal function and no evidence of bone disease but exhibit continual decline in renal function and death by 20 weeks of age, when approximately $40 \%$ to $60 \%$ of them have vascular calcification. Temporal changes in serum parameters of JCK mice relative to wild-type mice from 6 through 18 weeks of age were shown to largely mirror serum changes commonly associated with clinical CKD-MBD. Bone histomorphometry revealed progressive changes associated with increased osteoclast activity and elevated bone formation [59].

Klotho null mice display premature aging and CKD-MBD-like phenotypes mediated by hyperphosphatemia and remediated by phosphate-lowering interventions (diets low in phosphate or vitamin D; knockouts of $1 \alpha$-hydroxylase, vitamin D receptor, or NaPi cotransporter) [11]. 


\subsection{Obstructive nephropathy}

The mouse with unilateral ureteral obstruction (UUO) is a well-established model of tubulointerstitial fibrosis of the kidney as the interstitial fibrosis is a hallmark of chronic renal failure [60]. We previously reported the vitamin D signaling attenuates renal fibrosis in obstructive nephropathy by suppressing the renin-angiotensin system (RAS) [61], furthermore, we found the mice developed hypocalcaemia and hyperparathyroidism after 7 days of ureteric obstruction [62], and the down-regulation of Cbfa1 and Col mRNA expression (Fig. 1) and the up-regulation of Tgf- $\beta$, CtsK, CaII, Opg and Rankl mRNA expression (Fig. 2) in tibia of UUO mice as well as the microarchitectural changes in the proximal tibia, likely to be precursors of the early stage during CKD-MBD [62]. The pathological alterations of proximal tibia in UUO group were characterized by a marked expansion of hypertrophic zone of chondrocytes and a dramatic decrease in osteoid content of the primary spongiosa zone, where the immature, poorly mineralized woven bones were present, indicating impaired mineralization of the newly formed bones (Fig. 3B). Above all, in addition to established genetic pathways, we suggest that the local skeletal renin-angiotensin system may be involved in the bone deteriorations associated with CKD as demonstrated by the marked up-regulation of protein expression of angiotensin II and its type 2 receptor in tibia of UUO mice (Fig. 4) [62].

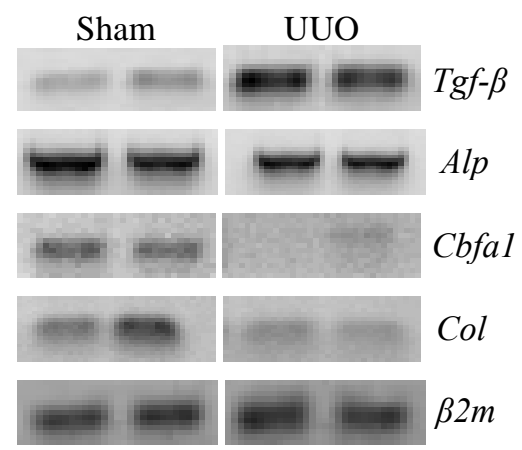

Figure 1. mRNA expression of osteoblast-specific genes in tibia of sham-operated and UUO mice

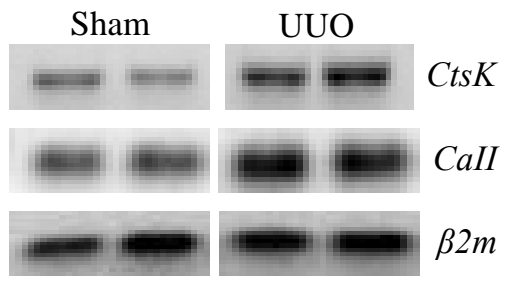

Figure 2. mRNA expression of osteoclast-specific genes in tibia of sham-operated and UUO mice 

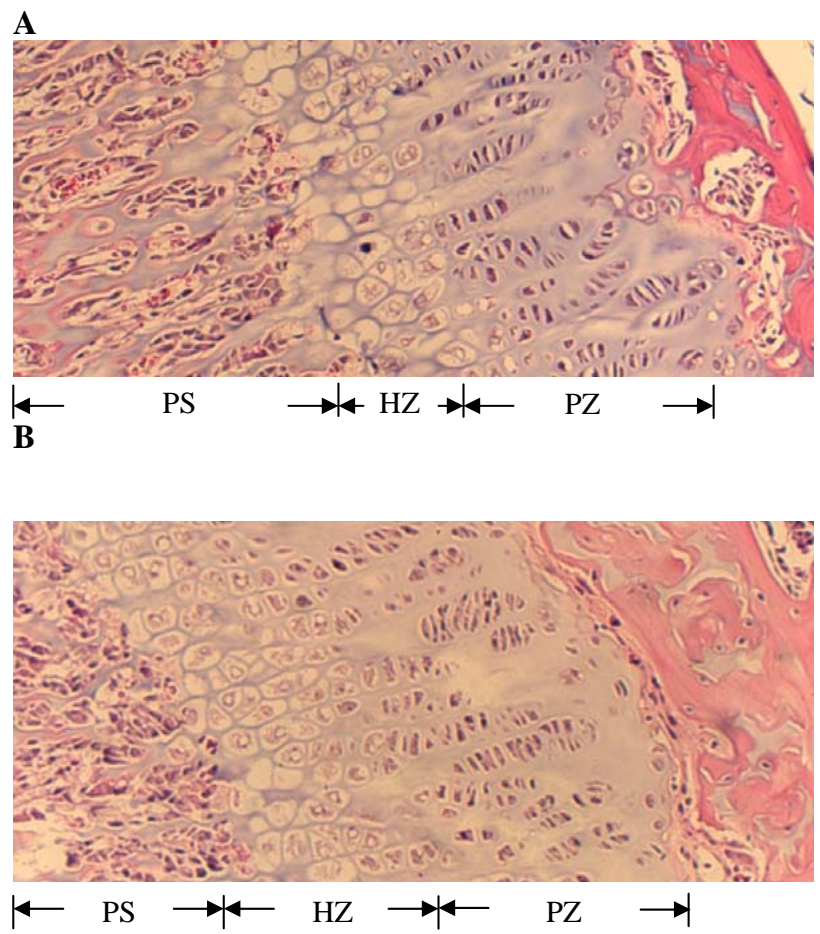

Figure 3. Hematoxylin and Eosin staining of the proximal tibia. The chondrocyte zone at growth plate was shown in A (Sham) and B (UUO) and it was visually separated into two areas, proliferative zone (PZ) and hypertrophic zone (HZ). Calcified cartilage with overlying newly bone underneath growth plate is known as the primary spongiosa (PS). Magnification, $\times 100$.

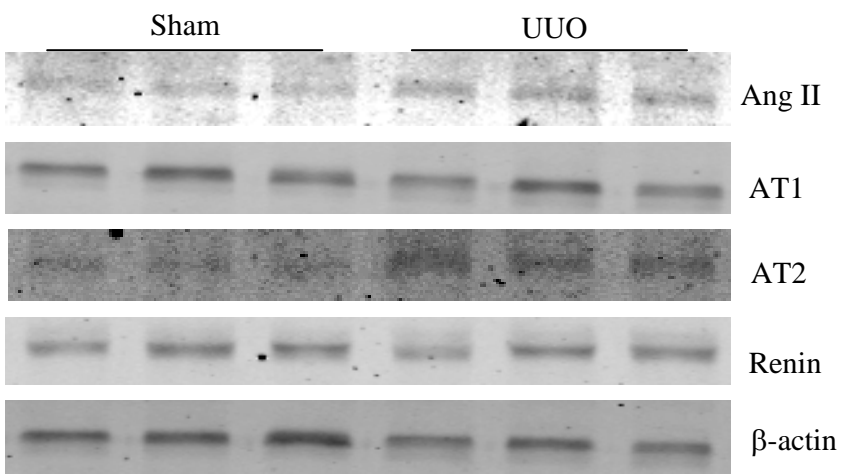

Figure 4. Protein expression of RAS components in mice tibia 


\section{Conclusion}

In summary, the present review demonstrates that the main pathological mechanisms involved in CKD-MBD are secondary hyperparathyroidism and vascular calcification. Therefore the main focus of the therapeutic research and the further molecular investigations should be on these main abnormalities associated with CKD. This can be achieved by employing the proper animal models for each of these complications including genetically modified mouse models. The animal models can play a great role in understanding the underlying mechanisms for CKD-MBD. The clinical treatment approaches should depend on the specific levels of the biomarkers of CKD patients. Further studies are needed to discover other pathological mechanisms for CKD-MBD other than those explained here. More advanced basic medical sciences should also be performed on the research and development of novel drugs with less adverse effects.

This work was sponsored by Shanghai Pujiang Program (10PJ1407700) and Innovation Program of Shanghai Municipal Education Commission (11ZZ137) for Yan Zhang.

\section{Author details}

Yan Zhang ${ }^{1,2^{*}}$ and Yoseph Asmelash Gebru ${ }^{1}$

*Address all correspondence to: medicineyan@yahoo.com.cn

1 Center for Systems Biomedical Sciences, University of Shanghai for Science and Technology, Shanghai , P.R.China

2 Department of Applied Biology and Chemical Technology, The Hong Kong Polytechnic University, Hung Hom, Kowloon, Hong Kong, P.R.China

\section{References}

[1] Mejía N, Roman-García P, Miar AB, Tavira B, Cannata-Andía JB. Chronic Kidney Disease - Mineral and Bone Disorder: A Complex Scenario. Nefrologia 2011;31(5) 514-519.

[2] Anca G, Stuart MS. Role of Vitamin D Deficiency in Chronic Kidney Disease. J Bone Miner Res 2007;22(S2) S91-94.

[3] Nickolas TL, Stein E, Cohen A, Thomas V, Staron RB, McMahon DJ, Leonard MB, Shane E. Bone Mass and Microarchitecture in CKD Patients with Fracture. J Am Soc Nephrol 2010;21(8) 1371-1380. 
[4] Chauhan V, Kelepouris E, Chauhan N, Vaid M. Current Concepts and Management Strategies in Chronic Kidney Disease-Mineral and Bone Disorder. South Med J 2012;105(9) 479-485.

[5] Tomasello S. Secondary Hyperparathyroidism and Chronic Kidney Disease. Diabetes Spectrum 2008;21(1) 19-25.

[6] Schumock GT, Andress DL, Marx SE, Sterz R, Joyce AT, Kalantar-Zadeh K. Association of Secondary Hyperparathyroidism with CKD Progression, Health Care Costs and Survival in Diabetic Predialysis CKD Patients. Nephron Clin Pract 2009;113(1) 54-61.

[7] Goodman WG. Medical Management of Secondary Hyperparathyroidism in Chronic Renal Failure. Nephrol Dial Transplant 2003;18(S3) 2-8.

[8] Kiattisunthorn K, Moe SM. Chronic Kidney Disease-Mineral Bone Disorder (CKDMBD). IBMS BoneKEy 2010;7(12) 447-457.

[9] Riggs MM, Peterson MC, Gastonguay MR. Multiscale Physiology-Based Modeling of Mineral Bone Disorder in Patients with Impaired Kidney Function. J Clin Pharmacol 2012;52(1) 45-53.

[10] Lips P. Vitamin D Deficiency and Secondary Hyperparathyroidism in the Elderly: Consequences for Bone Loss and Fractures and Therapeutic Implications. Endocr Rev 2001;22(4) 477-501.

[11] Kuro-O M. Phosphate and Klotho. Kidney Int Suppl 2011;(121) S20-23.

[12] Komaba H. CKD-MBD (Chronic Kidney Disease-Mineral and Bone Disorder). Role of FGF23 Klotho Axis in CKD-MBD. Clin Calcium 2010;20(7) 1028-1036.

[13] Rodríguez M, López I, Muñoz J, Aguilera-Tejero E, Almaden Y. FGF23 and Mineral Metabolism, Implications in CKD-MBD. Nefrologia 2012;32(3) 275-278.

[14] Moe SM, Chen NX, Seifert MF, Sinders RM, Duan D, Chen X, Liang Y, Radcliff JS, White KE, Gattone VH 2nd. A Rat Model of Chronic Kidney Disease-Mineral Bone Disorder (CKD-MBD) and the Effect of Dietary Protein Source. Kidney Int 2009;75(2) 176-184.

[15] Vattikuti R, Towler DA. Osteogenic Regulation of Vascular Calcification: An Early Perspective. Am J Physiol Endocrinol Metab 2004;286(5) 686-696.

[16] Covic A, Kanbay M, Voroneanu L, Turgut F, Serban DN, Serban IL, Goldsmith DJ. Vascular Calcification in Chronic Kidney Disease. Clin Sci 2010;119(3) 111-121.

[17] Shao JS, Cai J, Towler DA. Molecular Mechanisms of Vascular Calcification: Lessons Learned From the Aorta. Arterioscler Thromb Vasc Biol 2006;26(7) 1423-1430.

[18] Proudfoot D, Shanahan CM. Molecular Mechanisms Mediating Vascular Calcification: Role of Matrix Gla Protein. Nephrology 2006;11(5) 455-461. 
[19] Speer MY, McKee MD, Guldberg RE, Liaw L, Yang HY, Tung E, Karsenty G, Giachelli CM. Inactivation of the Osteopontin Gene Enhances Vascular Calcification of Matrix Gla Protein-Deficient Mice: Evidence for Osteopontin as an Inducible Inhibitor of Vascular Calcification In Vivo. J Exp Med 2002;196(8) 1047-1055.

[20] Shanahan CM, Proudfoot D, Farzaneh-Far A, Weissberg PL. The Role of Gla Proteins in Vascular Calcification. Crit Rev Eukaryot Gene Expr 1998;8(3-4) 357-75.

[21] Kidney Disease: Improving Global Outcomes (KDIGO) CKD-MBD Work Group. KDIGO Clinical Practice Guideline for the Diagnosis, Evaluation, Prevention and Treatment of Chronic Disease -Mineral and Bone Disorder (CKD-MBD). Kidney Int 2009;(113) S1-130.

[22] Morrone LF, Russo D, Di Iorio B. Diagnostic Workup for Disorders of Bone and Mineral Metabolism in Patients with Chronic Kidney Disease in the Era of KDIGO Guidelines. Int J Nephrol DOI: 10.4061/2011/958798

[23] Gao P, D'Amour P. Evolution of the Parathyroid Hormone (PTH) Assay--Importance of Circulating PTH Immunoheterogeneity and of its Regulation. Clin Lab 2005;51(1-2) 21-29.

[24] Kidney Disease: Improving Global Outcomes (KDIGO) CKD-MBD Work Group. KDIGO Clinical Practice Guideline for the Diagnosis, Evaluation, Prevention, and Treatment of Chronic Kidney Disease-Mineral and Bone Disorder (CKD-MBD). Kideny Int Suppl 2009;76(113) S1-130.

[25] Hu MC, Shi M, Zhang J, Quiñones H, Griffith C, Kuro-o M, Moe OW. Klotho Deficiency Causes Vascular Calcification in Chronic Kidney Disease. J Am Soc Nephrol 2011;22(1) 124-136.

[26] Jiang JQ, Lin S, Xu PC, Zheng ZF, Jia JY. Serum Osteoprotegerin Measurement for Early Diagnosis of Chronic Kidney Disease-Mineral and Bone Disorder. Nephrology 2011;16(6) 588-594.

[27] Ford ML, Smith ER, Tomlinson LA, Chatterjee PK, Rajkumar C, Holt SG. FGF-23 and Osteoprotegerin Are Independently Associated With Myocardial Damage in Chronic Kidney Disease Stages 3 and 4. Another Link between Chronic Kidney Disease-Mineral Bone Disorder and the Heart. Nephrol Dial Transplant 2012;27(2) 727-733.

[28] Bacchetta J, Boutroy S, Juillard L, Vilayphiou N, Guebre-Egziabher F, Pelletier S, Delmas PD, Fouque D. Bone Imaging and Chronic Kidney Disease: Will High-Resolution Peripheral Tomography Improve Bone Evaluation and Therapeutic Management? J Ren Nutr 2009;19(1) 44-49.

[29] Bacchetta J, Boutroy S, Vilayphiou N, Juillard L, Guebre-Egziabher F, Rognant N, Sornay-Rendu E, Szulc P, Laville M, Delmas PD, Fouque D, Chapurlat R. Early Impairment of Trabecular Microarchitecture Assessed with HR-pQCT in Patients with Stage II-IV Chronic Kidney Disease. J Bone Miner Res 2010;25(4) 849-857. 
[30] Raggi P, Bellasi A. Clinical Assessment of Vascular Calcification. Adv Chronic Kidney Dis 2007;14(1) 37-43.

[31] Martin KJ, González EA. Long-term Management of CKD-Mineral and Bone Disorder. Am J Kidney Dis 2012;60(2) 308-315.

[32] Martin KJ, González EA. Prevention and Control of Phosphate Retention/Hyperphosphatemia in CKD-MBD: What is Normal, When to Start, and How to Treat? Clin J Am Soc Nephrol 2011;6(2) 440-446.

[33] Negi S, Shigematsu T. CKD-MBD (Chronic Kidney Disease-Mineral and Bone Disorder). Lanthanum Carbonate and New Phosphate Binders in Patients with Chronic Kidney Disease. Clin Calcium 2010;20(7) 1096-1102.

[34] Cozzolino M, Mazzaferro S, Brandenburg V. The Treatment of Hyperphosphataemia in CKD: Calcium-Based or Calcium-Free Phosphate Binders? Nephrol Dial Transplant 2011;26(2) 402-407.

[35] Kazama JJ. Oral Phosphatebinders: History and Prospects. Bone 2009;45(S1) S8-12.

[36] Spaia S. Phosphate Binders: Sevelamer in the Prevention and Treatment of Hyperphosphataemia in Chronic Renal Failure. Hippokratia 2011;15(1) 22-26.

[37] Frazão JM., Adragão T. Non-Calcium-Containing Phosphate Binders: Comparing Efficacy, Safety, and other Clinical Effects. Nephron Clin Pract 2012;120(2) 108-119.

[38] Ogata H, Koiwa F, Kinugasa E, Akizawa T. CKD-MBD: Impact on Management of Kidney Disease. Clin Exp Nephrol 2007;11(4) 261-268.

[39] Barreto FC, de Oliveira RA, Oliveira RB, Jorgetti V. Pharmacotherapy of Chronic Kidney Disease and Mineral Bone Disorder. Expert Opin Pharmacother 2011;12(17) 2627-2640.

[40] Wüthrich RP, Martin D, Bilezikian JP. The Role of Calcimimetics in the Treatment of Hyperparathyroidism. Eur J Clin Invest 2007;37(12) 915-922.

[41] Bover J, Aguilar A, Baas J, Reyes J, Lloret MJ, Farré N, Olaya M, Canal C, Marco H, Andrés E, Trinidad P, Ballarin J. Calcimimetics in the Chronic Kidney Disease-Mineral and Bone Disorder. Int J Artif Organs 2009;32(2) 108-121.

[42] Bacchetta J, Harambat J, Cochat P, Salusky IB, Wesseling-Perry K. The Consequences of Chronic Kidney Disease on Bone Metabolism and Growth in Children. Nephrol Dial Transplant 2012;27(8) 3063-3071.

[43] Mitu G, Hirschberg R. Bone Morphogenetic Protein-7 (BMP7) in Chronic Kidney Disease. Front Biosci 2008;13 4726-39.

[44] González EA, Lund RJ, Martin KJ, McCartney JE, Tondravi MM, Sampath TK, Hruska KA. Treatment of a Murine Model of High-Turnover Renal Osteodystrophy by Exogenous BMP-7. Kidney Int 2002;61(4) 1322-1331. 
[45] White AP, Vaccaro AR, Hall JA, Whang PG, Friel BC, McKee MD. Clinical Applications of BMP-7/OP-1 in Fractures, Nonunions and Spinal Fusion. Int Orthop 2007;31(6) 735-741.

[46] Jha V, Kher V, Pisharody R, Sharma RK, Abraham G, Gokulnath, Almeida A, Gupta A. Indian Commentary on the 2009 KDIGO Clinical Practice Guideline for the Diagnosis, Evaluation, and Treatment of Chronic Kidney Disease-Mineral and Bone Disorders. Indian J Nephrol 2011;21(3) 143-151.

[47] Tominaga Y, Matsuoka S, Sato T. Surgical Indications and Procedures of Parathyroidectomy in Patients with Chronic Kidney Disease. Ther Apher Dial 2005;9(1) 44-47.

[48] Onoda N, Kashiwagi T, Nakamura T, Niitsu Y, Omata M, Kurihara S. Parathyroid Interventions for Secondary Hyperparathyroidism in Hemodialyzed Patients. Ther Apher Dial 2005;(S1) S11-15.

[49] Jofré R, López Gómez JM, Menárguez J, Polo JR, Guinsburg M, Villaverde T, Pérez Flores I, Carretero D, Rodríguez Benitez P, Pérez García R. Parathyroidectomy: Whom and When? Kidney Int Suppl 2003;(85) S97-100.

[50] Naranda J, Ekart R, Pečovnik-Balon B. Total Parathyroidectomy with Forearm Autotransplantation as the Treatment of Choice for Secondary Hyperparathyroidism. J Int Med Res 2011;39(3) 978-987.

[51] Cunningham J, Locatelli F, Rodriguez M. Secondary Hyperparathyroidism: Pathogenesis, Disease Progression, and Therapeutic Options. Clin J Am Soc Nephrol 2011;6(4) 913-921.

[52] Sinanoglu O, Sezgin G, Ozturk G, Tuncdemir M, Guney S, Aksungar FB, Yener N. Melatonin with 1,25-Dihydroxyvitamin D3 Protects against Apoptotic Ischemia-Reperfusion Injury in the Rat Kidney. Ren Fail 2012;34(8) 1021-1026.

[53] Shobeiri N, Adams MA, Holden RM. Vascular Calcification in Animal Models of CKD: A Review. Am J Nephrol 2010;31(6) 471-481.

[54] Padagas J, Colloton M, Shalhoub V, Kostenuik P, Morony S, Munyakazi L, Guo M, Gianneschi D, Shatzen E, Geng Z, Tan HL, Dunstan C, Lacey D, Martin D. The Receptor Activator of Nuclear Factor-Kappab Ligand Inhibitor Osteoprotegerin is a Bone-Protective Agent in a Rat Model Of Chronic Renal Insufficiency and Hyperparathyroidism. Calcif Tissue Int 2006;78(1) 35-44.

[55] Iwasaki Y, Kazama JJ, Yamato H, Fukagawa M. Changes in Chemical Composition of Cortical Bone Associated with Bone Fragility in Rat Model with Chronic Kidney Disease. Bone 2011;48(6) 1260-1267.

[56] Neven E, D'Haese PC. Vascular Calcification in Chronic Renal Failure: What Have We Learned from Animal Studies? Circ Res 2011;108(2) 249-264.

[57] Wahner HW. Measurements of Bone Mass and Bone Density. Endocrinol Metab Clin North Am 1989;18(4) 995-1012. 
[58] Price PA, Roublick AM, Williamson MK. Artery Calcification in Uremic Rats is Increased by a Low Protein Diet and Prevented by Treatment with Ibandronate. Kidney Int 2006;70(9) 1577-1583.

[59] Sabbagh Y, Graciolli FG, O'Brien S, Tang W, Dos Reis LM, Ryan S, Phillips L, Boulanger J, Song W, Bracken C, Liu S, Ledbetter S, Dechow P, Canziani ME, Carvalho AB, Jorgetti V, Moyses RM, Schiavi SC. Repression of Osteocyte Wnt/B-Catenin Signaling is an Early Event in the Progression of Renal Osteodystrophy. J Bone Miner Res 2012;27(8) 1757-1772.

[60] Zhang Y, Wu SY, Gu SS, Lv FK. Changes of Renal Vitamin D Metabolic Enzyme Expression and Calcium Transporter Abundance in Obstructive Nephropathy. Nephrology 2011;16(8) 710-714.

[61] Zhang Y, Kong J, Chang A, Deb DK, Li YC. Vitamin D Receptor Attenuates Renal Fibrosis in Obstructive Nephropathy by Suppressing the Renin-Angiotensin System. J Am Soc Nephrol 2010;21(6) 966-973.

[62] Gu SS, Zhang Y, Wu SY, Diao TY, Gebru YA, Deng H. Early Molecular Responses of Bone to Obstructive Nephropathy Induced by Unilateral Ureteral Obstruction in Mice. Nephrology 2012;17(8) 767-773 